\title{
Posibilidades del análisis de imagen para el estudio de la sintesis de materiales por combustión
}

\author{
E. CHINARRO', B. MORENO', D. MARTÍN², L. GONZÁLEZ², E. VILLANUEVA², D. GUINEA², J.R. JURADO' \\ 'Instituto de Cerámica y Vidrio (CSIC). Cantoblanco, Madrid, España. \\ ${ }^{2}$ Instituto de Automática Industrial (CSIC). Arganda del Rey, Madrid, España
}

\begin{abstract}
El análisis de la imagen obtenida por una cámara de video digital se utiliza para el estudio de la reacción de síntesis por combustión de $\mathrm{Sm}_{0.95} \mathrm{CoO}_{3-\delta}$. El método de combustión presenta como característica intrínseca la generación de materiales nanométricos cerámicos y metal-cerámicos; permitiendo la producción de compuestos con actividad catalítica. En este trabajo se obtienen polvos de tamaño de cristalito entorno a $5 \mathrm{~nm}$, con una superficie específica de $24 \mathrm{~m}^{2} / \mathrm{g}$ y como consecuencia bastante reactivos. Se llevaron cabo estudios preliminares sobre la etapa de ignición de la síntesis usando el análisis de imagen por descomposición de los planos de los tres colores primarios; dichos experimentos muestran que la llama generada en esta etapa de ignición es un proceso termodinámicamente aleatorio. La finalidad de este trabajo es establecer un protocolo de experimentación para la futura correlación entre los parámetros de la síntesis por combustión de un material, naturaleza y concentración de precursores entre otros, y la distribución de temperatura durante la ignición, con objeto de optimizar y controlar el proceso. Se abre una línea de investigación basada en la utilización de la técnica de análisis de imagen para realizar un seguimiento controlado de la evolución de la temperatura e intensidad de la llama durante la etapa de ignición de la síntesis por combustión de un material.
\end{abstract}

Palabras clave: sintesis de materiales por combustión, análisis de imagen, temperatura de llama, planos de intensidad.

\section{Study of material combustion synthesis by image analysis}

Analysis of the recorded image by a digital video camera of the $\mathrm{Sm}_{095} \mathrm{CoO}_{3-\delta}$ combustion synthesis is carried out. Intrinsically the combustion method yields nanometric materials (ceramic and metal-ceramic); allowing to produce catalytic powders. In this work, nanocrystalline powders of around $5 \mathrm{~nm}$ with $24 \mathrm{~m}^{2} / \mathrm{g}$ of specific surface area and, consequently, high reactivity powders were obtained. Preliminary studies were done about ignition step of the synthesis, using image analysis from the three primary colours planes decomposition, these experiments show that the generated flame in this ignition step is a thermodynamically random process. The final aim of this work is to establish a protocol of performance to a future correlation among relevant combustion synthesis parameters of materials, i.e. the precursors nature and concentration, and the temperature distribution during the ignition, to optimise and control the process. This investigation opens an important field, the use of image analysis to carry out a thorough study of the temperature evolution and of the flame intensity during the ignition step of a material combustion synthesis.

Keywords: materials combustion synthesis, image analysis, flame temperature, intensity planes.

\section{INTRODUCCIÓN}

La síntesis por combustión de materiales avanzados (cerámicos, metales y cermets) es un método de síntesis de reciente desarrollo $(1,2)$ y la evaluación del comportamiento térmico durante todo el proceso es complicado.

Una alternativa para conocer la evolución de temperatura durante la ignición, es la captura y análisis de la secuencia de imágenes de la muestra a lo largo del proceso. El análisis de imagen permite determinar la distribución de radiación de las reacciones de combustión con la debida resolución espacial y temporal, frente a otros sistemas como el pirómetro óptico y el termopar, ambos puntuales y de lenta respuesta, que no permiten medirla.

\subsection{Síntesis por combustión}

En 1967 (3), con el descubrimiento del fenómeno de la llama sólida o proceso de combustión en estado sólido, el método de combustión comenzó a implantarse como método de síntesis alternativo a las vías tradicionales. La síntesis por combustión es un método rápido, fiable y eficaz para obtener polvos cerámicos, metálicos, vidrios y cermets. En este proceso se utiliza como fuente energética el calor liberado en la reacción a partir de los reactivos, dando lugar a un proceso autosostenido. En algunos casos es preciso activar el proceso, ya que la entalpía de la reacción de síntesis no es suficiente para lograr que el proceso se autopropague. Se puede realizar de varias formas, una de las cuales es la adición 
de un combustible que pueda oxidarse con los reactivos de la síntesis.

La termodinámica que rige la síntesis por combustión surge inicialmente del campo de los propulsores y explosivos. La mezcla combustible-oxidante se expresa en términos de razón de la mezcla, razón de equivalencia $(\phi)$ o coeficiente estequiométrico de elementos $\left(\phi_{\mathrm{e}}\right)$. Barrere (4), en 1960, definió el carácter de la mezcla en cuanto al combustible según la razón de equivalencia $(\phi)$. En 1968, Bakhman (5) introduce el concepto de coeficiente estequiométrico de los elementos $\left(\phi_{\mathrm{e}}\right)$, en el que tiene en cuenta la relación entre el combustible y el oxidante intramolecular. De manera que, cuando la mezcla es estequiométrica $\phi$ y $\phi_{\mathrm{e}}$ son iguales a la unidad y en caso contrario dichos valores pueden variar bastante entre sí, aunque ambos mantengan la misma tendencia, ya que $\phi, \phi_{\mathrm{e}}<1$ si la mezcla es rica en combustible y $\phi, \phi_{\mathrm{e}}>1$ si es pobre en dicho combustible.

En 1981, Jain y col. (6) propusieron un método muy sencillo para calcular la cantidad de combustible necesario para preparar una mezcla estequiométrica $\left(\phi_{\mathrm{e}}=1\right)$ [Ec.1]:

\section{$\sum$ (coef. elem. reductores $\times$ valencia $)+$ $\sum($ coef, elem, oxidantes $\times$ valencia $)=0$}

considerando la valencia que presentan los elementos en los gases desprendidos como productos de la combustión $\left(\mathrm{CO}_{2^{\prime}}\right.$ $\mathrm{H}_{2} \mathrm{O}, \mathrm{N}_{2}$ ), es decir, $\mathrm{C}+4, \mathrm{H}+1, \mathrm{O}-2$ y $\mathrm{N} 0$.

La elección del combustible es importante debido a que la exotermicidad de la combustión varía con el combustible, modificando con ello las características de los productos obtenidos (7). Los combustibles más utilizados son el tetraformato de triazina (TFTA, $\mathrm{C}_{4} \mathrm{H}_{16} \mathrm{~N}_{6} \mathrm{O}_{2}$ ) (8), hidrazidas como la hidrazida maleica $\left(\mathrm{C}_{4} \mathrm{H}_{4} \mathrm{~N}_{2} \mathrm{O}_{2}\right)$ y la carbohidrazida $\left(\mathrm{CO}\left(\mathrm{N}_{2} \mathrm{H}_{3}\right)_{2}\right)$ (9), la sacarosa $\left(\mathrm{C}_{14} \mathrm{O}_{12} \mathrm{H}_{20}\right)$ y la carbamida, más conocida cómo urea $\left(\mathrm{CON}_{2} \mathrm{H}_{4}\right)(1,2,9-15)$. Todos estos compuestos difieren entre sí por su poder reductor y por la cantidad de gases que liberan durante la combustión, siendo estos dos factores determinantes tanto de las condiciones de reacción, como por ejemplo la temperatura de la llama, como de la morfología y composición (porosidad, sinterización prematura, estructuras más o menos cristalinas, etc.) de los productos obtenidos.

La urea es uno de los combustibles que se emplean con mayor frecuencia. Se trata de un compuesto de bajo coste económico y que, al liberar menor cantidad de gases, suministra temperaturas de llama más altas que otros combustibles (2).

Otro factor importante a considerar es la naturaleza de los precursores de los cationes metálicos que intervienen en la reacción. Se suelen utilizar nitratos hidratados porque poseen un marcado carácter oxidante que ayuda a superar la barrera de energía de activación de la reacción, y porque funden a bajas temperaturas garantizando con ello una mezcla homogénea a una temperatura inferior a la de descomposición del combustible. Sin embargo, en aquellas ocasiones en las que no es posible encontrar un nitrato hidratado, es necesario recurrir a otro tipo de sal. En estos casos se añade a la mezcla de reacción nitrato de amonio $\left(\mathrm{NH}_{4} \mathrm{NO}_{3}\right)$ como oxidante adicional (12), que no modifica la temperatura de reacción pero sí incrementa la cantidad de gases liberados, dando lugar a estructuras mucho más esponjosas y con una mayor superficie específica.
Por último es importante señalar que cada material presenta unas condiciones óptimas de temperatura y tiempo de reacción, y la transformación de fase intrínseca es fija y característica de cada sistema. Por ello, el aumento en la eficiencia de la síntesis debe quedar sometido a una adecuada gestión del calor en el proceso de combustión y a la reducción de pérdidas de calor del medio, por convección y radiación.

Algunos autores han introducido modificaciones en el sistema de aporte de calor para iniciar la combustión, como por ejemplo el uso de una mufla precalentada a $500{ }^{\circ} \mathrm{C}$ para que la combustión tenga lugar en el interior de la misma a una temperatura constante en todos sus puntos $(12,14)$, o la utilización de un microondas en sustitución de la placa calefactora (16-19); en ambos casos se obtiene mayor homogeneidad durante el calentamiento, pero se sigue sin saber la temperatura que alcanza la llama.

Por otro lado, la medida de la temperatura de la llama durante la combustión se ha intentado llevar a cabo utilizando un termopar $(10,16)$ colocado en el interior del contenedor donde se produce la reacción, pero se trata de una medida puntual que no ofrece una información real sobre la temperatura de la combustión en el área total del proceso).

\subsection{Fundamentos del análisis de imagen.}

El ojo humano detecta los colores como combinaciones variables de tres colores: rojo, verde y azul (colores primarios). La CIE (Commission Internationale de l'Éclairage) $(20,21)$ asignó en 1931 los siguientes valores de longitudes de onda a los colores primarios: azul $(435,8 \mathrm{~nm})$, verde $(546,1 \mathrm{~nm})$ y rojo (700nm).

Así, el análisis de imagen se centra en el modelo RGB (Red Green Blue) que consiste en obtener tres planos de color (plano rojo, plano verde y plano azul) a partir de la radiación emitida por la escena grabada en la cámara de vídeo; mediante la ponderación de estos tres planos se obtiene la imagen a color (22).

La imagen se adquiere por un sensor (plano fotosensible de photosites (elementos semiconductores excitados por fotones) (23)) que recoge el haz incidente. Posteriormente en su representación digital en el PC, se descompone en tres matrices de intensidades (planos de píxeles (superficie homogénea más pequeña de los que componen una imagen) o planos de color). El haz rojo descompuesto del haz incidente, formará el plano de color rojo, el haz verde el plano de color verde y el haz azul el plano de color azul.

Cada valor de la matriz esta definido en un intervalo de valores [0-255], dependiendo de la intensidad luminosa que haya recibido ese photosite, por lo tanto, la intensidad luminosa recibida en el photosite es directamente proporcional al valor del píxel ([0-255]) correspondiente en la matriz de intensidades o plano de color. De esta forma se obtienen tres planos de color, que muestran directamente las intensidades de radiación recibida en el rojo, verde y azul de la escena observada.

Además, los sensores pueden presentar fundamentos tecnológicos diferentes, CCD (Charge Coupled Device) (24) o CMOS (Complementary Metallic Oxide Semiconductor) (25). La primera tecnología produce una señal continua de video y consta de una matriz de $\mathrm{M}^{\star} \mathrm{N}$ photosites. La tecnología CMOS, más moderna, ofrece más integración, bajo consumo y mayor sensibilidad (26).

La imagen tratada en el PC se presenta digitalizada espacialmente en forma de matriz con una resolución de 
$M^{\star} N$ píxeles. Cada plano de color se refiere a una función de intensidad bidimensional $f_{R}(x, y), f_{V}(x, y)$ y $f_{A}(x, y)$, donde $x$ e $\mathrm{y}$ son las coordenadas espaciales y el valor de $\mathrm{f}$ en cualquier punto $(x, y)$ es proporcional a la intensidad o nivel de gris de la imagen en ese punto.

En resumen, medir la temperatura de la llama durante la combustión resulta muy complicado por la rapidez del proceso y por la falta de homogeneidad de la misma. Las posibilidades de aplicación del análisis de imagen para determinar la temperatura de la llama durante la combustión, consisten por un lado en utilizar la combinación de los tres planos de color para medir la temperatura del sistema (asociar "color de imagen" con "temperatura del color"), y por otro lado, estudiar cada plano de color por separado, es decir, reanalizar los planos de intensidad en el estudio de la homogeneidad del sistema; ambas posibilidades se detallan en el apartado de análisis e interpretación de imagen.

El objetivo del presente artículo es realizar una primera aproximación al estudio, y el seguimiento de la evolución térmica del proceso de ignición mediante el análisis de imagen, en la síntesis por combustión del material $\mathrm{Sm}_{0.95} \mathrm{CoO}_{3-\delta}$.

\section{PROCEDIMIENTO EXPERIMENTAL}

\subsection{Materiales y síntesis por combustión de $\mathrm{Sm}_{0.95} \mathrm{CoO}_{3-\delta}$}

Los precursores utilizados para la síntesis por combustión del la cerámica $\mathrm{Sm}_{0.95} \mathrm{CoO}_{3-\delta}$ fueron los siguientes, $\mathrm{Sm}\left(\mathrm{NO}_{3}\right)_{3} \cdot 6 \mathrm{H}_{2} \mathrm{O}$ (Aldrich, $\left.99.9 \%\right), \mathrm{Co}\left(\mathrm{NO}_{3}\right)_{3} \cdot 6 \mathrm{H}_{2} \mathrm{O}$ (Merck, 99 $\%)$, y urea $\left(\mathrm{CO}\left(\mathrm{NH}_{2}\right)_{2}\right)$ (Aldrich, $98 \%$ ) como combustible.

La mezcla de los precursores necesarios, según la composición a preparar, se realizó en una cápsula de sílice vítrea, con un diámetro inferior de $14 \mathrm{~cm}$ y un diámetro superior de $14.5 \mathrm{~cm}$. De este modo, existe una amplia superficie de contacto de la mezcla con el aire, garantizando el oxígeno necesario para que la combustión ocurra. Esta mezcla se calienta (en placa calefactora) a baja temperatura, entre 100 y $150{ }^{\circ} \mathrm{C}$, temperatura suficiente para que se fundan los precursores (nitratos y la urea) y se forme una disolución, dando lugar a una perfecta homogeneización de los precursores, en esta etapa también se produce la eliminación del agua de hidratación. A continuación, se eleva la temperatura, para favorecer la reacción de combustión, hasta aproximadamente $300{ }^{\circ} \mathrm{C}$, temperatura a la cual la disolución comienza a hervir. La ebullición, cada vez más violenta, da lugar a un desprendimiento masivo de gases y la ignición tiene lugar, manifestándose de manera instantánea mediante una llama. El producto final que se obtiene presenta aspecto esponjoso, y se muele fácilmente. Todo este proceso no tarda más de 5 minutos y el esquema del mismo viene representado en la figura 1.

Durante la etapa de ignición existe proyección de polvos, con la consecuente pérdida de los mismos. Para evitar esta proyección se ha utilizado un exceso del $100 \%$ de urea. Con esta cantidad de combustible se ha conseguido reducir las pérdidas por proyección, permitiendo obtener un rendimiento de la reacción de un 95-97\%.

Se realizó la calcinación del producto obtenido a 500 ${ }^{\circ} \mathrm{C}$ durante $12 \mathrm{~h}$, con una rampa de calentamiento de $5{ }^{\circ} \mathrm{C} /$ min, para obtener la fase cristalina del material. Los polvos obtenidos directamente de la combustión y los calcinados fueron caracterizados mediante difracción de rayos $\mathrm{X}$,

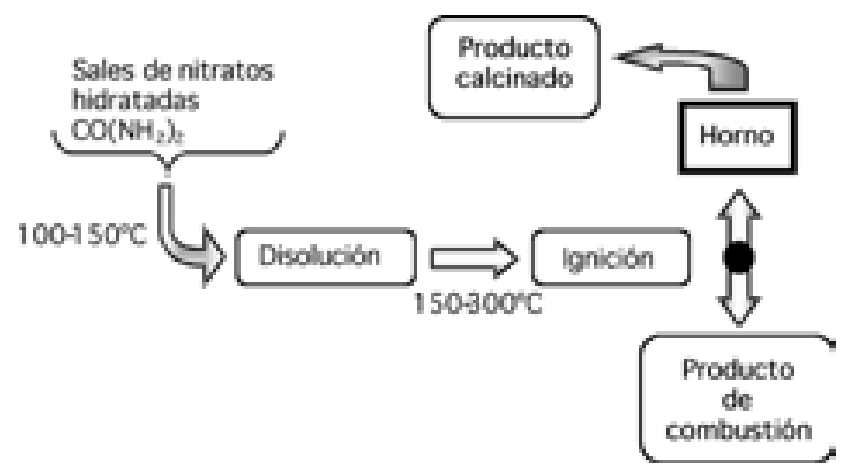

Fig. 1.- Esquema del proceso de combustión que se ha utilizado para preparar las muestras.

en un difractómetro Siemens mod. D5000 con generador Kristalloflex 710, para observar si presentaba alguna fase cristalina y realizar su identificación estructural (programa EVAApplication V6.0) por comparación con los difractogramas almacenados en la base de datos PDF-2 Database sets 1-46. Se utilizó la microscopía electrónica de barrido (microscopio Zeiss-DSM 950) y de transmisión (microscopio JEOL 2010), para determinar la morfología y el tamaño de partícula que presenta el polvo obtenido. Para hallar la superficie específica del producto de la combustión se usó un equipo Monosorb Surface Area Analyzer (Quantachrome Corporation, mod. MS-13) y se aplicó el modelo de isoterma BET.

\subsection{Procedimiento experimental de análisis de imagen.}

El experimento se realizó colocando una cámara de video digital SONY MAVICA MVC-CD300 (MPEG video, 24-bit PAL, 320*240 píxeles, sensor CCD de color $8.3 \mathrm{~mm}$ (tipo $1 / 1.8)$ ) formando un ángulo de aproximadamente $45^{\circ}$ con respecto a la base de la combustión, como se indica en la figura 2. Por las características de absorción espectral de los dispositivos CCD realizados en Silicio, la cámara es sensible a la radiación del IR próximo y a la región del visible. La visión nocturna (cámaras "cero lux") se consigue suprimiendo los filtros ópticos que eliminan el IR, para permitir sensibilidad del sistema a longitudes de onda superiores al rojo, de gran interés en este estudio.

Antes de realizar la grabación se eliminaron las influencias de las fuentes de radiación externas (luz solar y fluorescentes) con el objetivo de captar únicamente la radiación procedente de la reacción de combustión. Para ello se situó la muestra en una zona oscura recubierta de material de baja reflectancia, libre de toda fuente de emisión y de los reflejos externos. Una vez instalado el equipo, se realizó la grabación de la combustión.

Para realizar el análisis de la imagen se seleccionó inicialmente un fotograma (frame) de la secuencia de vídeo digital con el programa THUMBS (27), a continuación se descompuso en los tres planos de color, obteniendo de cada uno de ellos su plano de intensidad; todo ello se procesó con el programa de cálculo MATLAB (28). 


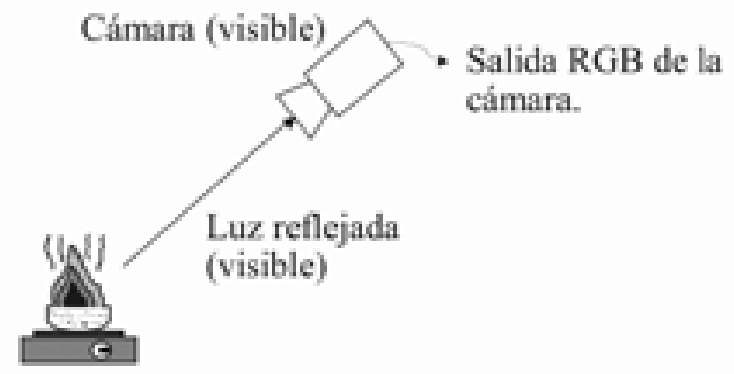

Reacción de combustión

Fig. 2.- Caracterización de la temperatura en una reacción de combustión, utilizando radiación del visible.

\section{RESULTADOS Y DISCUSIÓN}

\subsection{Síntesis de los materiales}

En la síntesis de este material por combustión se obtiene un polvo muy homogéneo, de tamaño nanométrico, y con baja cristalinidad como indica el difractograma de rayos $X$ (figura 3).

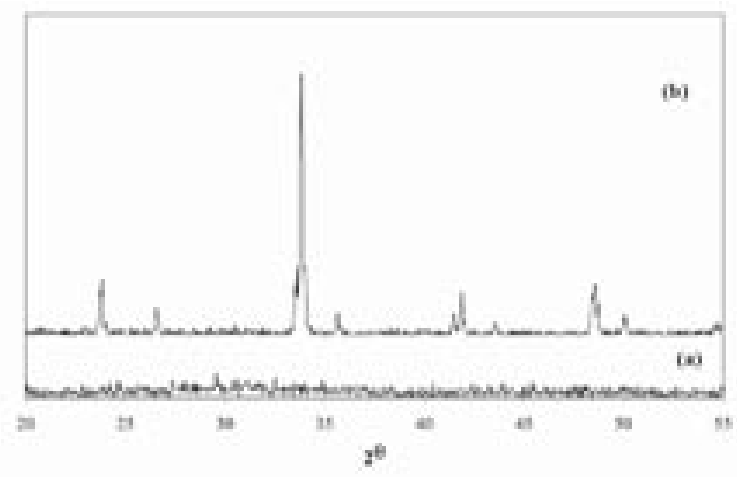

Fig. 3.- Difracción de rayos $\mathrm{X}$ de los polvos de $\mathrm{SmCoO}_{3-\delta}$ (a) obtenidos directamente por combustión y (b) calcinados a $500{ }^{\circ} \mathrm{C} / 12 \mathrm{~h}$.

Después de un minucioso estudio de identificación estructural, y teniendo en cuenta la rapidez del proceso de combustión, el resultado proporcionado por los rayos X parece mostrar que lo que se obtiene directamente de la combustión es, para este compuesto, además de las trazas de precursores (combustible y nitratos) y productos de su descomposición, la mezcla de los óxidos de los cationes metálicos precursores, es decir, los óxidos de samario y de cobalto. Estos óxidos presentan alta reactividad, puesto que calcinando la muestra a $500{ }^{\circ} \mathrm{C}$ se consigue eliminar dichas trazas y obtener $\mathrm{SmCoO}_{3}$ cristalina monofásica, cuando esta fase se consigue, por otros métodos de síntesis, utilizando tratamientos térmicos por encima de los $900{ }^{\circ} \mathrm{C}(29)$.

La posterior caracterización del producto de reacción revela que está formado por aglomerados interconectados entre sí que forman un entramado poroso con aspecto esponjoso y frágil (figura 4(a)). Se comprobó que la muestra era nanocristalina, con un tamaño medio de cristalito de aproximadamente $5 \mathrm{~nm}$, observado por microscopía de transmisión (figura 4 (b)). El valor de superficie específica, determinada por BET, obtenido fue de $24 \mathrm{~m}^{2} / \mathrm{g}$.

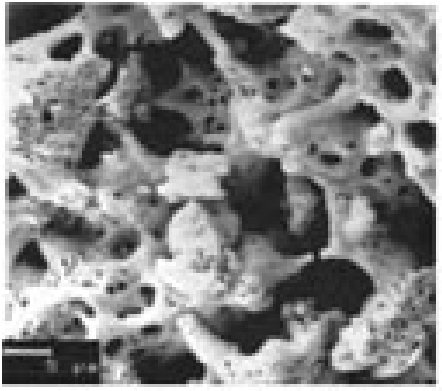

(a)

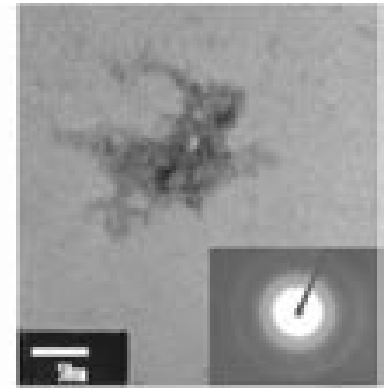

(b)
Fig. 4.- Micrografías de MEB (a), MET y difracción de electrones (b) del polvo $\mathrm{Sm}_{0.95} \mathrm{CoO}_{3-\delta}$ de combustión (sin tratar).

Según los estudios previos realizados en síntesis por combustión de otros sistemas (2) y los resultados obtenidos de la caracterización de los polvos por difracción de rayos X (figura 3), las reacciones que probablemente tienen lugar durante la síntesis por combustión son las siguientes:

R1.

$$
\begin{array}{r}
\mathrm{Sm}\left(\mathrm{NO}_{3}\right)_{3} \cdot 6 \mathrm{H}_{2} \mathrm{O}(\mathrm{c}) \rightarrow 1 / 2 \mathrm{Sm}_{2} \mathrm{O}_{3}(\mathrm{c})+3 / 2 \mathrm{~N}_{2}(\mathrm{~g})+15 / 4 \mathrm{O}_{2}(\mathrm{~g})+6 \mathrm{H}_{2} \mathrm{O}(\mathrm{g}) \\
\Delta \mathrm{H}^{\circ}=\sim+155.40 \mathrm{Kcal} / \mathrm{mol}
\end{array}
$$

R2. ${ }^{*}$

$\mathrm{Co}\left(\mathrm{NO}_{3}\right)_{3} \cdot 6 \mathrm{H}_{2} \mathrm{O}(\mathrm{c}) \rightarrow 5 / 8 \mathrm{CoO}(\mathrm{c})+1 / 8 \mathrm{C}_{3} \mathrm{O}_{4}(\mathrm{c})+3 / 2 \mathrm{~N}_{2}(\mathrm{~g})+63 / 16 \mathrm{O}_{2}(\mathrm{~g})+6 \mathrm{H}_{2} \mathrm{O}(\mathrm{g})$ $\Delta \mathrm{H}^{\mathrm{o}}=+118.98 \mathrm{Kcal} / \mathrm{mol}$

R3.

$\left(\mathrm{CO}\left(\mathrm{NH}_{2}\right)_{2}\right)(\mathrm{c})+3 / 2 \mathrm{O}_{2}(\mathrm{~g}) \rightarrow \mathrm{CO}_{2}(\mathrm{~g})+2 \mathrm{H}_{2} \mathrm{O}(\mathrm{g})+\mathrm{N}_{2}(\mathrm{~g})$ $\Delta \mathrm{H}^{\mathrm{o}}=-129.90 \mathrm{Kcal} / \mathrm{mol}$

RT.

$\mathrm{Sm}\left(\mathrm{NO}_{3}\right)_{3} \cdot 6 \mathrm{H}_{2} \mathrm{O}(\mathrm{c})+\mathrm{Co}\left(\mathrm{NO}_{3}\right)_{3} \cdot 6 \mathrm{H}_{2} \mathrm{O}(\mathrm{c})+\mathrm{m}\left(\mathrm{CO}\left(\mathrm{NH}_{2}\right)_{2}\right)(\mathrm{c})+(3 / 2 \mathrm{~m}-123 / 16) \mathrm{O}_{2}(\mathrm{~g}) \rightarrow$

$1 / 2 \mathrm{Sm}_{2} \mathrm{O}_{3}(\mathrm{c})+5 / 8 \mathrm{CoO}(\mathrm{c})+1 / 8 \mathrm{CO}_{3} \mathrm{O}_{4}(\mathrm{c})+\mathrm{mCO}_{2}(\mathrm{~g})+(3+\mathrm{m}) \mathrm{N}_{2}(\mathrm{~g})+(12+2 \mathrm{~m}) \mathrm{H}_{2} \mathrm{O}(\mathrm{g})$ $\Delta \mathrm{H}^{\mathrm{o}}=(\sim+274.40-129.90 \mathrm{~m}) \mathrm{Kcal} / \mathrm{mol}$

${ }^{*}$ ) siendo esta reacción (R2) mucho más probable que:

$\mathrm{Co}\left(\mathrm{NO}_{3}\right)_{3} \cdot 6 \mathrm{H}_{2} \mathrm{O} \rightarrow 1 / 2 \mathrm{Co}_{2} \mathrm{O}_{3}+3 / 2 \mathrm{~N}_{2}+15 / 4 \mathrm{O}_{2}+6 \mathrm{H}_{2} \mathrm{O}$

puesto que el $\mathrm{Co}_{2} \mathrm{O}_{3}$ descompone según la reacción:

$\mathrm{Co}_{2} \mathrm{O}_{3} \rightarrow 5 / 4 \mathrm{CoO}+1 / 4 \mathrm{Co}_{3} \mathrm{O}_{4}+3 / 8 \mathrm{O}_{2}$

en el intervalo $890-930^{\circ} \mathrm{C}(30)$, temperaturas que se superan en la combustión.

Las variaciones de entalpía implicadas en estas reacciones químicas se han calculado a partir de las entalpías de formación a $25^{\circ} \mathrm{C}$ publicadas en la ref. (31), lo mismo que los datos en los que se ha basado el cálculo estimado de la entalpía de reacción 
de R1, puesto que no se encontró en la literatura el dato de $\Delta \mathrm{H}_{\mathrm{f}}^{\mathrm{o}}$ del Sm $\left.\left(\mathrm{NO}_{3}\right)_{3} \cdot 6 \mathrm{H}_{2} \mathrm{O}(\mathrm{c})\right)$. La reacción de combustión de la urea (R3) es exotérmica y debe proporcionar el calor necesario para la reacción de síntesis. Las reacciones individuales de formación de los óxidos a partir de los nitratos hidratados (R1 y R2) son endotérmicas. Utilizando la cantidad de urea necesaria para obtener una mezcla estequiométrica respecto a especies oxidantes y especies reductoras $\left(\phi_{\mathrm{e}}=1\right)(6), \mathrm{m}=$ 4.04 moles de urea, la reacción total (RT) es exotérmica $\left.\left(\Delta \mathrm{H}^{\mathrm{o}}=-250.40\right) \mathrm{Kcal} / \mathrm{mol}\right)$, ya que se utiliza más combustible de lo que sería necesario para que la reacción RT fuese termodinámicamente estequiométrica $(\mathrm{m}=2.11$ moles urea). A todo este calor desprendido de las reacciones se le suma el calor extra suministrado por el exceso de urea (100\%) que se ha utilizado $\left.\left(\Delta \mathrm{H}^{\circ}=-775.19\right) \mathrm{Kcal} / \mathrm{mol}\right)$. Este carácter exotérmico de la reacción provoca el calentamiento del sistema y con ello una elevación de la temperatura que termina con la ignición del mismo. Como resultado de esta auto-ignición se produce una llama capaz de alcanzar temperaturas superiores a los $1000^{\circ} \mathrm{C}$.

\subsection{Análisis e interpretación de imagen.}

La reacción de combustión consta de tres etapas bien diferenciadas las cuales sirven de base al estudio del procedimiento experimental de análisis de imagen:

\section{A. PRIMER INTERVALO de TEMPERATURA: $0^{\circ}-300{ }^{\circ} \mathrm{C}$}

El material comienza a calentarse y emite radiación en el IR lejano, la combustión avanza en el combustible produciendo sustancias grisáceas, gases no inflamables, vapor de agua y calor (el combustible empieza a reaccionar sin llama). La radiación emitida por la reacción de combustión en esta etapa cumple la ley de radiación del cuerpo negro (32), es decir, la distribución espectral de radiación es continua y tiene un máximo dependiente de la temperatura para una determinada longitud de onda o frecuencia de radiación. Introduciendo la longitud de onda del máximo del espectro de la radiación del cuerpo negro a la ley de desplazamiento de Wien [Ec.2]:

$\lambda_{\max } \cdot \mathrm{T}=2.898 \cdot 10^{-3} \mathrm{~m} \cdot \mathrm{K}$

A medida que aumenta la temperatura $\mathrm{T}$, el máximo se desplaza hacia longitudes de onda menores (mayores frecuencias). Así, utilizando esta relación y una cámara de IR se puede determinar la temperatura de la reacción de combustión. La cámara de vídeo digital proporciona el máximo de radiación (la máxima aparición de un determinado nivel de gris corresponde a la longitud de onda en la que aparece el máximo de intensidad) y, por lo tanto, se mide la longitud de onda del máximo, $\lambda_{\max }$ a la cual el cuerpo negro emite con intensidad máxima; aplicando posteriormente la ley de desplazamiento de Wien se calcula la temperatura. El nivel de gris que refleja el máximo de intensidad se obtiene realizando un histograma de la imagen, (el histograma de una imagen es una función discreta que representa el número de píxeles en la imagen en función de los niveles de intensidad).

Alternativamente al proceso anterior, calculando la integral del histograma (área bajo la curva del histograma que corresponde a la intensidad total captada) de la imagen y aplicando la ley de Stefan- Boltzmann [Ec. 3] se podría obtener la temperatura global de la reacción de combustión.

La ley establece que la intensidad total, $\mathrm{Wm}^{-2}$, de la radiación emitida por un cuerpo negro para todo el espectro electromagnético es:

$\mathrm{W}=\sigma \mathrm{T}^{4}$

siendo $\sigma=5.670 \cdot 10^{-8}\left(\mathrm{Wm}^{-2} \mathrm{~K}^{-4}\right)$.

\section{B. IGNICIÓN, SEGUNDO INTERVALO de} TEMPERATURA: $300^{\circ}-1000^{\circ} \mathrm{C}$ :

Un cuerpo negro en este intervalo de temperaturas emite radiación en el IR, por lo que solo se puede detectar esta radiación con una cámara termográfica con un sensor de IR lejano sensible a radiación térmica.

TABla I. Porcentaje de la CONTRIBUCiÓN DE ENERGía EMITIDA EN FORMA DE RADIACIÓN INFRARROJA, VISIBLE Y ULTRAVIOLETA, EN LA RADIACIÓN DE UN CUERPO NEGRO A DIFERENTES TEMPERATURAS (32).

\begin{tabular}{|c|c|c|c|}
\hline $\begin{array}{c}\text { Temperatura } \\
(\mathrm{K})\end{array}$ & $\begin{array}{c}\% \\
\text { infrarrojo }\end{array}$ & $\%$ visible & $\begin{array}{c}\% \\
\text { ultravioleta }\end{array}$ \\
\hline 1000 & 99.999 & $7.367^{\star} 10^{-4}$ & $3.258^{\star} 10^{-11}$ \\
\hline 2000 & 98.593 & 1.406 & $7.4000^{\star} 10^{-4}$ \\
\hline 3000 & 88.393 & 11.476 & 0.131 \\
\hline
\end{tabular}

Observando los datos mostrados en la tabla I se deduce que, a baja temperatura $(1000 \mathrm{~K})$ prácticamente toda la radiación es infrarroja, mientras que al aumentar la temperatura se incrementa la contribución de energía emitida en la banda del espectro visible disminuyendo en la zona infrarroja.

Aproximadamente a $300{ }^{\circ} \mathrm{C}$ el calor desprendido es muy grande y el combustible desprende gases inflamables por efecto del calor, estos arden y generan llama (figura 5). A partir de este punto crítico no se puede calcular la temperatura de la combustión siguiendo la ley de radiación del cuerpo negro, y se procede a estimar la temperatura utilizando el concepto de temperatura de color (33-37). Ésta se establece por asociación con una llama típica o por la caracterización de la llama a analizar en la región visible del espectro electromagnético.

El color de la llama está directamente relacionado con los combustibles y comburentes que reaccionan y la temperatura generada por dicha reacción (38). El color es consecuencia de la distribución de energía electromagnética originada por la llama en el espectro visible. Para establecer una relación consistente entre la composición cromática de una imagen y la temperatura de cada punto es preciso eliminar la influencia del resto de los factores involucrados, fijando la naturaleza y composición de los elementos que originan la combustión así como otras fuentes de radiación ajenas al proceso en estudio. De esta forma podemos establecer una correlación aproximada entre la temperatura de cada punto de la imagen y la distribución espectral de energía, los planos de color, de la misma.El método empírico de temperatura de color eintensidad permite estudiar la llama de la reacción de combustión para la preparación de $\mathrm{Sm}_{0.95} \mathrm{CoO}_{3-\delta}$ (figura 5). En dicha figura se muestran los tres planos de color de la imagen de la llama, se aprecia claramente como el sensor capta más radiación en la región roja (figura 5(a)) que en las regiones verde (figura 5(b)) y azul (figura 5(c)) del espectro visible, lo que implica que la energía emitida en la región de baja frecuencia (rojo) es superior a la que emite en frecuencias superiores (verde y azul). En correspondencia con las temperaturas de la combustión de las sustancias involucradas si la temperatura de una región se 
eleva aparece un desplazamiento hacia frecuencias superiores, incrementándose el área correspondiente al plano de color que representa energías superiores por fotón (azul).

Teniendo en cuenta las reacciones propuestas en el apartado 3.1, se pueden determinar los gases liberados en cada reacción. Los gases liberados son $\mathrm{N}_{2}, \mathrm{O}_{2}, \mathrm{CO}_{2}$ y $\mathrm{H}_{2} \mathrm{O}$, cada uno de los cuales producirá una radiación electromagnética de acuerdo con las transiciones electrónicas de las especies involucradas en función de la temperatura que alcance la reacción.

Como consecuencia de los ensayos realizados para establecer un patrón experimental entre las variables de imagen adquirida y la evolución de la distribución espacial de la temperatura durante el proceso se propone el siguiente protocolo de experimentación:

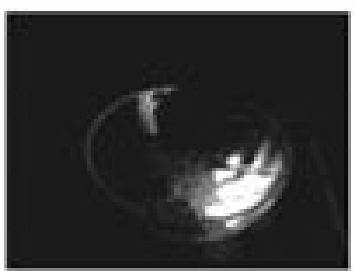

(a)

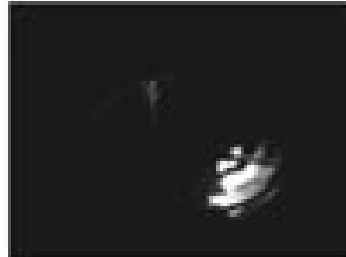

(b)

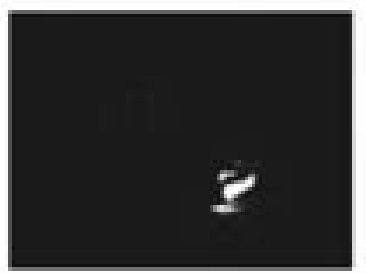

(c)

Fig. 5.- Planos de color rojo (a), verde (b) y azul (c), de la imagen de la llama en un instante de la reacción de combustión en estudio.

i) establecimiento de la relación existente entre el color de una llama conocida, procedente de una fuente de referencia a una determinada temperatura, y el color obtenido con una cámara de video digital en condiciones similares,

ii) calibración de la temperatura asociada al color de la llama, en una combustión de urea y nitrato amónico con una temperatura característica (patrón óptico),

iii) utilización de un termógrafo óptico para caracterizar las temperaturas de las distintas llamas que aparecen en la reacción de combustión (patrón óptico),

iv) observación de la evolución de homogeneidad en la reacción de combustión, a partir de las intensidades captadas por cada plano de color.

Además del estudio de los planos de color y su relación con la temperatura de la llama en un cierto instante, este procedimiento permite el estudio de la evolución de las intensidades de energía electromagnética emitida en cada banda del espectro y en cada punto de la imagen a lo largo del proceso. Este método empírico se desarrolló en los tres planos de color obteniéndose, debido a la radiación emitida por las llamas en la etapa de 300 a $1000{ }^{\circ} \mathrm{C}$, la mayor cantidad de energía emitida y, por tanto, la mejor observación de la distribución espacial de intensidades en el plano rojo. Un ejemplo de dicha evolución se ilustra en la figura 6 (representación tridimensional de una imagen instantánea de la distribución de energía de la banda espectral del rojo en el proceso de combustión).

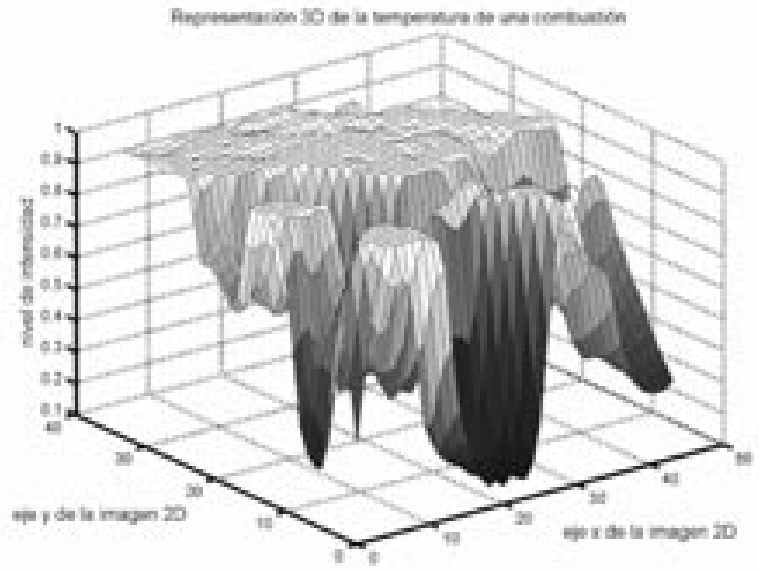

Fig. 6.- Representación 3D de una región del plano de intensidades en la zona del espectro electromagnético correspondiente al rojo (figura $5(a))$.

El plano x-y representa el área de la imagen, el eje z de la gráfica representa la intensidad de cada píxel [0-255], y aparece normalizado en el intervalo [0-1]. Se han utilizado diferentes niveles de gris en la representación para indicar con negro las zonas de menor intensidad, que corresponden a las paredes de la cápsula (mayor transferencia de calor), gris para las zonas de intensidad intermedia y finalmente blanco para representar las zonas de mayor intensidad que están asociadas a las llamas.

El estudio inicial del análisis de imagen nos aporta información sobre la evolución intensidad-tiempo. Con el objetivo de demostrar esta afirmación se ha analizado en el plano rojo el video completo del "frame" de la figura 5(a), así, se muestra la evolución del centro de masas (CDM) de la zona de máxima intensidad de la llama en esta banda espectral (figura 7(a)) y evolución del área de la zona de máxima intensidad de la llama (figura $7(b)$ ).

La figura 7 muestra aleatoriedad del CDM e inestabilidad de la distribución de energía visible en el plano rojo (es decir, inestabilidad de la temperatura).

Como resultado de este estudio preliminar se puede afirmar que dicho método permite el registro y cuantificación de la distribución espacio-temporal de la temperatura de un proceso termodinámicamente aleatorio como es una llama. En esta primera aproximación se detectan tres asociaciones posibles entre la imagen obtenida por una cámara de video digital y una reacción de combustión. La primera relativa a la correspondencia entre los tres planos de color (imagen en color), y el patrón térmico bidimensional que corresponde a esa imagen, para caracterizar en función de la temperatura, los colores de las llamas que se producen en este tipo de combustiones en la etapa de 300 a $1000^{\circ} \mathrm{C}$. La segunda estudia cada plano de color (planos de intensidades), y analiza la evolución espacio-temporal de intensidad por separado para 
Evolucion del CDM de la zona de maxima intensidad
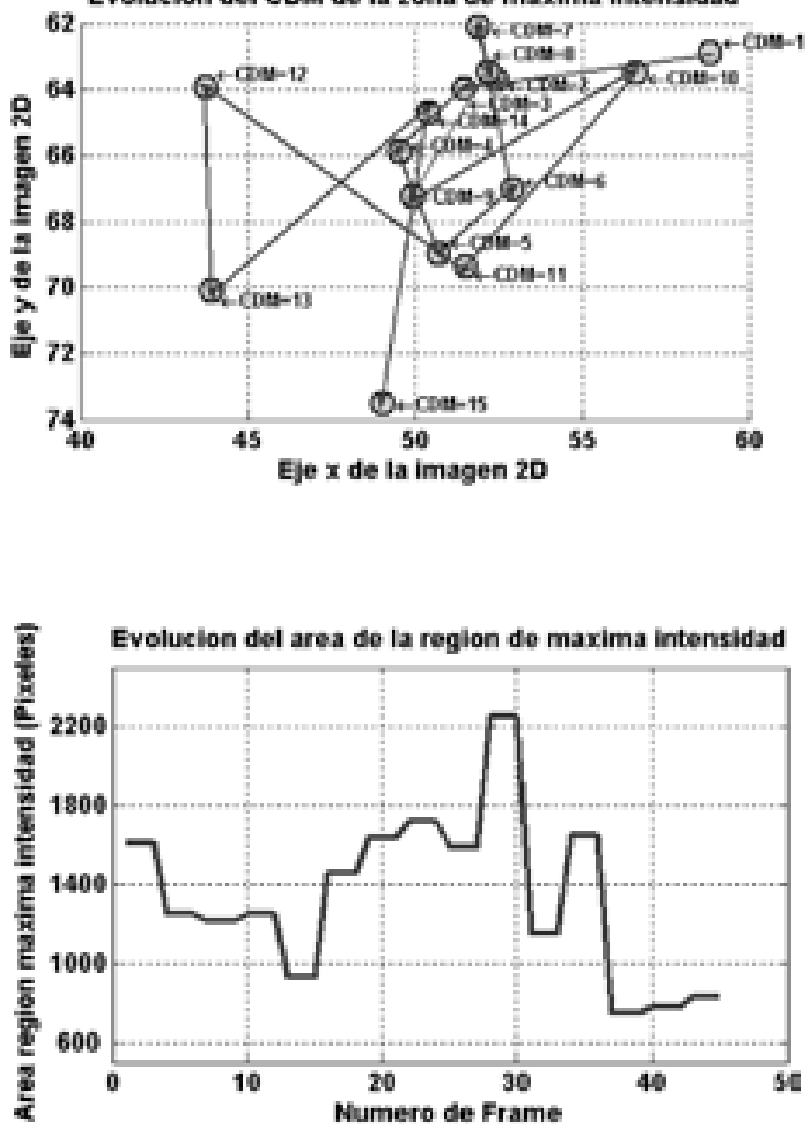

Fig. 7.- Evolución del centro de masas (a) y área (b) de la zona de máxima intensidad de la llama en la banda de emisión correspondiente al rojo del espectro.

investigar la distribución de emisión espectral en la banda correspondiente a cada color en el proceso de combustión. Y la tercera consistiría en un estudio computacional que permita la determinación geométrica del proceso de combustión en el entorno espacio-tiempo-intensidad.

A todo esto se le une el hecho de la sencillez y el bajo coste del método en comparación con los convencionales como la termografía.

\section{ENFRIAMIENTO}

Posteriormente a la desaparición de la llama debido al enfriamiento, el estudio es similar al de la primera etapa (estudio basado en radiación IR)

\section{CONCLUSIONES}

1. Se muestra en este trabajo que mediante la síntesis por combustión se obtienen polvos nanométricos, homogéneos, de baja cristalinidad, alta superficie específica y alta reactividad. Se trata de una síntesis rápida, donde se obtienen llamas que alcanzan temperaturas superiores a $\operatorname{los} 1000^{\circ} \mathrm{C}$.

2. En este trabajo el análisis de imagen es una técnica apropiada para el estudio experimental del proceso de combustión. Permite el seguimiento de la evolución desde la etapa de ignición, con la debida resolución temporal y espacial, visualizando la distribución de temperaturas durante el proceso de síntesis.

3. El método utilizado permite establecer una correlación entre los parámetros ópticos de la imagen -distribución de intensidades en cada banda de color- y la distribución de temperatura durante la ignición, con objeto de controlar y optimizar el proceso.

\section{AGRADECIMIENTOS}

Los autores de este trabajo agradecen la financiación de los contratos Post Doctoral I3P-CSIC, y a los proyectos CICYT MAT2001-1489-CO2-02, APOLLON ENK5-CT-2001-00572 y ACERINOX.

\section{REFERENCIAS}

1. D.A. Fumo, M.R. Morelli, A.M. Segadães. "Combustion Synthesis of Calcium Aluminates" Mater. Res. Bull. 31 [10], 1243-1255, (1996)

2. D.A. Fumo, J.R. Jurado, A.M. Segadães, J.R. Frade. "Combustion synthesis of iron substituted strontium titanate perovskites" Mater. Res. Bull. 32 [10], 1459-1470, (1997)

3. Z.A. Munir, J.B. Holt. "The combustion synthesis of refractory nitrides. 1. Theoretical-Analysis" J. Mater. Sci. 22 (2), 710-714 (1987)

4. M. Barrere, A. Jaumotte, B.F. DeVeubeke, J. Vandenkerekhove. "Rocket Propulcsion" 132-134, Ed. Elsevier, Amsterdam, 1960

5. N.N. Bakhman. Combust. Explo. Shock Waves 4:9, (1968)

6. S.R. Jain, K.C. Adiga, V.R. Pai Verneker. "A New Approach to Thermochemical Calculations of Condensed Fuel-Oxidizer Mixtures". Combustion and flame 40, 71-79 (1981)

7. S.S. Manoharan, K. C. Patil. “Combustion Synthesis of Metal Chromite Powders". J. Am. Ceram. Soc. 75 [4], 1012-1015 (1992)

8. S.S. Manoharan, N.R.S. Kumar, K.C. Patil. "Preparation of fine particle chromites: a combustion approach". Mat. Res. Bull. 25, 731-738 (1990)

9. J.J. Kingsley, K. Suresh, K.C. Patil. “Combustion synthesis of fine-particle metal aluminates" J. Mater. Sci. 25, 1305-1312 (1990)

10. S. Bhaduri, S.B. Bhaduri, K.A. Prisbrey. "Auto ignition synthesis of nanocrystalline $\mathrm{MgAl}_{2} \mathrm{O}_{4}$ and related nanocomposites". J. Mater. Res., 14 [9], 3571-3580 (1999)

11. A.C. Tas. "Chemical Preparation of the Binary Compounds in the CalciaAlumina System by Self-Propagating Combustion Synthesis". J. Am. Ceram. Soc. 81 [11], 2853-2863 (1998)

12. A.M. Segadães, M.R. Morelli, R.G.A. Kiminami. “Combustion Synthesis of Aluminium Titanate" J. Eur. Ceram. Soc. 18, 771-781 (1998)

13. S. Bhaduri, E. Zhou, S.B. Bhaduri. "Combustion synthesis of nanocrystalline powders in $\mathrm{Al}_{2} \mathrm{O}_{3}-\mathrm{MgO}$ binary system". Ceram. Eng. Sci. Proceed., 18 [4], 645-652 (1997)

14. S. Bhaduri, E. Zhou, S.B. Bhaduri. "Autoignition processing of nanocrystlline $\alpha-\mathrm{Al}_{2} \mathrm{O}_{3}$ " NanoStruct. Mater. 7[5], 487-496 (1996)

15. E. Breval, K.K. Agrawal. "Syntheis of [NZP]-Structure-Type Materials by the Combustion Reaction Method". J. Am. Ceram. Soc. 81 [7], 1729-1735 (1998)

16. X. Zeng, G. Sun, S. Zang. “Combustion synthesis of $\mathrm{Al}_{2} \mathrm{O}_{3}\left(-\mathrm{Cr}_{2} \mathrm{O}_{3}\right)-\mathrm{Cr}$ cermets". Script. Mater. 42, 1167-1172 (2000)

17. V. Chandramouli, S. Anthonysamy, P.R.V. Rao. "Combustion synthesis of thoria - a feasibility study". J. Nuclear Mater. 265, 255-261 (1999)

18. S. Anthonysamy, K. Ananthasivan, V. Chandramouli, I. Kaliappan, P.R.V. Rao. "Combustion synthesis of urania-thoria solid solutions". J. Nuclear Mater. 278, 346-357 (2000)

19. Y.P. Fu, Ch.H. Lin. "Microwave-induced combustion synthesis of $\mathrm{Ni}^{-} \mathrm{Zn}$ ferrite powder and its characterization" J. Magnetism and Magnetic Materials 251 [1], 74-79 (2002)

20. CIE History 1913-1988, Publication CIE 82-1990, ISBN 3900734194

21. Fundamental Radiometry and Photometry, Eastman Kodak Company, Image Sensor Solutions, Rochester, New York 14650, February 7, 2000

22. Visión por Computador. Imágenes digitales y aplicaciones, Gonzalo Pajares, Jesús M. De la Cruz, Editorial Ra-Ma

23. Solid State Image Sensors. Terminology, Eastman Kodak Company, Microelectronic Technology Division, Rochester, New York 14650-2010, December 8, 1994

24. Charge-Coupled Device (CCD) Image Sensors, Eastman Kodak Company, Image Sensor Solutions, Rochester, New York 14650-2010, May 29, 2001

25. Shutter Operations for CCD and CMOS Image Sensor, Eastman Kodak Company, Image Sensor Solutions, December 17, 2003 
26. E.N. Malanas, E.G.M. Petrakis, M. Zervakis, L. Petit, J-D. Legat. "A survay on industrial vision systems, applications and tools" Image and vision computing 21, 171-188 (2003)

27. Thumbs + Plus, User Guide Version 3.0. Cerious Software, Inc. Charlotte North Caroline, USA, 1996

28. Image Processing Toolbox, User's Guide Version 3.2, The MathWorks, Inc., July, 2002

29. H.Y. Tu, Y. Takeda, N. Imanishi, O. Yamamoto. "Ln $\mathrm{Sr} \mathrm{CoO}(\mathrm{Ln}=\mathrm{Sm}$ Dy) for electrode of solid oxide fuel cells". Solid State Ionics 100, 283-288 (1997)

30. Z. Nan, Q. Jiao, Z.Ch. Tan, S. Li-Xian. "Investigation of thermodynamic properties of $\mathrm{Co}_{2} \mathrm{O}_{3}$ powder". Thermochimica Acta 404, 245-249 (2003)

31. Lange's Handbook of Chemistry, 12th ed., Ed. J.A. Dean, McGraw-Hill, New UYork, 1979

32. P.K. Jain. "IR, visible and UV components in the spectral distribution of blackbody radiation". Phys. Educ. 31, 149-155 (1996)

33. Colorimetry, 2nd ed., CIE Publication 15.2, 1986
34. J. Fontecha, J. Campos, A. Corróns and A. Pons. "An analytical method for estimating correlated colour temperature uncertainty". Metrologia 39, 531-536 (2002)

35. J. L. Gardner. "Correlated colour temperature - uncertainty and estimation". Metrologia 37, 381-384 (2000)

36. J. F. Collins. "The colour temperature of daylight". Br. J. Appl. Phys. 16, 527-532 (1965)

37. A. R. Pearson, B. Pleasance. "The colour temperatures of the hefner and acetylene flames". Proc. Phys. Soc. 47, 1032-1041 (1935)

38. R. F. Barrow, E. F. Caldin. "Some Spectroscopic Observations on Pyrotechnic Flames". Proc. Phys. Soc. B 62, 32-39 (1949)

Recibido: 22.03 .04

Aceptado: 09.12.04 